Cahiers $d u$ MONDE RUSSE

\section{Cahiers du monde russe}

Russie - Empire russe - Union soviétique et États indépendants

$53 / 4 \mid 2012$

Varia

\title{
David El Kenz, François-Xavier Nérard, éds., Commémorer les victimes en Europe
}

\section{Françoise Mayer}

\section{OpenEdition}

\section{Journals}

Édition électronique

URL : http://journals.openedition.org/monderusse/7730

DOI : $10.4000 /$ monderusse. 7730

ISSN : $1777-5388$

Éditeur

Éditions de l'EHESS

\section{Édition imprimée}

Date de publication : 15 décembre 2012

ISSN : $1252-6576$

Référence électronique

Françoise Mayer, « David El Kenz, François-Xavier Nérard, éds., Commémorer les victimes en Europe » Cahiers du monde russe [En ligne], 53/4 | 2012, mis en ligne le 02 décembre 2013, Consulté le 22 septembre 2020. URL : http://journals.openedition.org/monderusse/7730 ; DOI : https://doi.org/ 10.4000/monderusse.7730

Ce document a été généré automatiquement le 22 septembre 2020.

(c) École des hautes études en sciences sociales 


\title{
David El Kenz, François-Xavier Nérard, éds., Commémorer les victimes en Europe
}

\author{
Françoise Mayer
}

\section{RÉFÉRENCE}

David EL KENZ, François-Xavier NÉRARD, éds., Commémorer les victimes en Europe, XVI $^{\mathrm{e}}$-XXI ${ }^{\mathrm{e}}$ siècles. Seyssel : Champ-Vallon, 2011, $352 \mathrm{p}$.

1 Sous ce titre, les auteurs de ce livre nous invitent à réfléchir à la présence des figures de victimes dans les manifestations de la mémoire collective en Europe, présence croissante depuis le $\mathrm{xvI}^{\mathrm{e}}$ siècle, au point que l'on puisse parler de «mémoire victimaire ".

2 C'est un fait, les mémoires collectives de nos sociétés contemporaines s'articulent dorénavant davantage autour des figures de victimes, que de celles de héros. Mais pour qu'il en soit ainsi, il a fallu que le regard porté sur la victime change radicalement au cours du temps. Ce qui nous parait banal aujourd'hui n'allait pas de soi avant la fin du XVIII ${ }^{\mathrm{e}}$ siècle, nous rappellent David El Kenz et François-Xavier Nérard dans l'introduction de cet ouvrage. Ce n'est en effet qu'à partir de cette époque, nous expliquent-ils, qu'apparaissent les premiers monuments célébrant le souvenir de victimes de luttes politiques, signes d'une évolution dans la manière de considérer les drames passés. Encore timide dans l'entre-deux-guerres, l'intérêt pour les victimes (civiles) de violences militaires ou politiques ne commence à se substituer à celui pour les héros qu'au début des années 1960, avec l'avènement de ce qu'Annette Wieviorka a appelé l'ère des témoins, en liaison avec le procès Eichmann à Jérusalem. Avant cette date, le rappel des faits héroïques et de résistance dominait largement la mémoire de la Seconde Guerre mondiale. Cette évolution est manifeste, particulièrement à partir des années 2000, avec l'instauration de commémorations et de mémoriaux aux victimes du 
judéocide qui constituent de toute évidence la matrice d'autres cultes victimaires, y compris dans l'Europe postcommuniste où les pratiques mémorielles concernent surtout les victimes du stalinisme et du Goulag, et marginalement celles du nazisme et de la Shoah.

Le rappel des victimes ne sert pas seulement la défense de valeurs abstraites et une construction identitaire, il s'inscrit aussi et avant tout dans un objectif de reconnaissance, souvent lié à la revendication très concrète de droits. Ceci comporte une part de stratégie, que les auteurs de cet ouvrage entendent interroger à partir de la conception des lieux de mémoire, telle que Pierre Nora l'a développée et testée dans sa monumentale entreprise collective du même nom à la fin du siècle dernier et qui met l'accent sur le volontarisme qu'implique tout travail mémoriel. En forgeant le concept de « lieu de mémoire victimaire », les auteurs ici réunis se proposent d'étudier des lieux qui ont pour vocation de cultiver le souvenir de victimes, et de le faire en s'attachant à comprendre tout à la fois leurs dimensions matérielle, symbolique et fonctionnelle.

L'ouvrage est issu d'un travail collectif dont les premiers résultats ont été présentés lors d'un colloque organisé à l'université de Bourgogne en novembre 2009. Il réunit les travaux de dix-huit auteurs, historiens ou politistes sur des champs temporels et géographiques très divers qui embrassent plusieurs siècles et une Europe qui va de l'Irlande à la Russie, en passant par l'Espagne. Ce rapprochement original d'expériences souvent très éloignées les unes des autres dans le temps ou dans l'espace permet de mieux saisir la façon dont les lieux de mémoire victimaire sont constitués, quand, où, par qui, pour qui, pour quoi, et avec quel effet social, étant entendu qu'un lieu de mémoire ne peut se comprendre en dehors du volontarisme qui, seul, peut l'instituer de façon pérenne. Les auteurs appuient leurs analyses sur des sources diversifiées, textuelles ou picturales. Tous s'interrogent sur ces lieux particuliers de mémoire en suivant des voies propres, non formatées, qui mettent l'accent selon le cas, sur des aspects différents du travail de mémoire aux différentes échelles sociales et nationales.

Plusieurs contributions éclairent la façon dont la notion de victime s'est imposée dans les représentations collectives, dans divers contextes historiques. Les auteurs travaillant sur des massacres très anciens, comme David El Kenz ou Karine Bigand, montrent comment la figure de victime doit surmonter les politiques d'oubliance qui étaient plutôt la règle à l'époque moderne. Ils s'attachent ensuite à retracer les destins de ces mémoires, en soulignant les enjeux culturels, confessionnels ou politiques qui entravent ou facilitent leur existence. Ludolf Pelizaeus, par exemple, explique comment les sorcières, considérées depuis le $\mathrm{xvI}^{\mathrm{e}}$ siècle comme d'horribles coupables, tombent dans l'oubli au siècle des Lumières, mais se parent d'un statut de victimes au XIX siècle à l'époque de l'essor du nationalisme allemand, sous l'effet d'un discours germaniste et anti-romain. Ce retournement de perspective sur les sorcières poursuivra son évolution entre les deux guerres, à la faveur tout à la fois d'un féminisme naissant, et d'un nationalisme germanique qui fera de ces victimes du fanatisme ecclésiastique un symbole de la nation aryenne. L'auteur analyse le poids de cet héritage historiographique sur les lieux censés cultiver la mémoire de figures toujours controversées du passé. Cet exemple est particulièrement éclairant sur la dimension plastique de la mémoire. D'autres auteurs montrent ce que le statut de victime peut avoir de fragile et en quoi son attribution n'est au fond jamais totalement acquise. JeanClément Martin nous invite à réfléchir à cette question à partir des victimes liées à l'histoire de la Révolution. Elles n'ont pas fait l'objet de mêmes cultes selon qu'elles 
sont "révolutionnaires ", "républicaines" ou "contre-révolutionnaires", et ne laissent donc pas les mêmes traces dans la mémoire et dans l'historiographie (l'une et l'autre étant plus à penser dans leur interaction que dans leur opposition). Karine Bigand, quant à elle, revient sur l'interprétation du soulèvement irlandais de 1641, un des rares événements de l'histoire britannique commémoré comme un massacre grâce à un travail de mémoire qui, depuis l'avènement des faits, a amplifié peu à peu le nombre des «massacrés» et des «victimes». Stéphane Michonneau s'intéresse au processus de victimisation dans la mémoire de la guerre civile espagnole et montre comment les contextes de guerre civile compliquent les assignations de statut de victimes, de bourreaux, d'agresseurs qui relèvent toujours de stratégies mémorielles, culturelles et politiques d'une grande complexité. En conclusion de l'ouvrage, Annette Becker opère un utile retour sur l'évolution de la notion de victime depuis les propositions conceptuelles de Lemkin, formulées à partir de 1942, et leurs fortunes diverses.

6 Au delà de la simple interrogation sur l'historicité de la notion de victime et ses possibles identités, c'est la question topographique des phénomènes mémoriels qui occupe les différents auteurs dans une riche palette de cas. Bien souvent, un lieu de mémoire s'institue sur le lieu même d'un massacre, ou bien sur celui où reposent les victimes, mais le lieu du souvenir peut être aussi «délocalisé ». Dans un cas comme dans l'autre, la mémoire s'est généralement transmise préalablement grâce à d'autres supports (littérature, peinture, témoignages privés ou judiciaires par exemple) qui peuvent plus ou moins peser sur la constitution d'un lieu de mémoire victimaire. Claire Gantet, dans son analyse des jeux de mémoire entre la guerre des Paysans (1525) et la guerre de Trente ans (1618-1648), s'intéresse aux supports rituels, architecturaux ou picturaux qui ont contribué à fixer ces représentations du passé. Ailleurs, dans le cas des « villages martyrs » de la Seconde Guerre mondiale, comme celui de Dun-les-Places qu'analyse Jean Vigreux, l'extension du statut de victime à l'ensemble d'une municipalité doit beaucoup à la logique judicaire d'après-guerre et aux politiques de reconnaissance tant à l'échelle locale que nationale, ce qui permet à cette notion de se cristalliser. Dans d'autres cas, comme celui que décrit Stéphane Michonneau, le village porte la marque de mémoires conflictuelles que les contemporains peuvent chercher à réconcilier, le lieu du souvenir est investi par des stratégies concurrentes qui peuvent se recouvrir selon les époques ou au contraire converger. Ailleurs encore, c'est à partir des tombes ou des fosses communes que peut s'instaurer une mémoire victimaire. C'est ce que montre Maurice Carrez à propos des vaincus de la guerre civile finlandaise qui seraient voués à l'oubli sans un activisme funéraire qui inscrit le souvenir des « rouges de 1918 » dans la topographie finlandaise, en dépit des obstacles politiques. Plusieurs auteurs questionnent la place de mémoriaux dans la mémoire collective et leur fonction. Comme François-Xavier Nérard qui observe comment, sur le site de la ville de Leningrad, le souvenir des victimes du blocus doit effacer celui des victimes de la Terreur stalinienne, respectivement honorés dans deux cimetières qui, à dessein, portent la même dénomination («cimetière mémorial»). Ou bien encore, Alexandra Goujon qui analyse une stratégie analogue, en Biélorussie, à partir du cas du complexe mémoriel de Hatin', censé rassembler l'hommage à toute une série de "villages martyrs " de la région et couvrir éventuellement la mémoire d'autres catégories de victimes, qu'il s'agisse des officiers polonais de Katyń (en Ukraine) ou encore des juifs supprimés en masse dans la région. Dans certains cas, la mémoire du massacre ne parvient pas à être cultivée sur le lieu même des faits, pour de multiples raisons. Le 
texte de David El Kenz explique ainsi comment la mémoire de la Saint-Barthélemy sera cultivée plutôt dans les Cévennes qu'à Paris, en dépit d'un certain activisme confessionnel. Taline Ter Minassian, quant à elle, analyse l'invention à Erevan, dans le contexte soviétique, d'un haut lieu de mémoire du génocide arménien, pour retracer l'histoire des sens qui lui sont assignés par la suite. Youri Shapoval montre comment un lieu de massacre de juifs pendant la guerre (Babij Jar, au nord-ouest de Kiev) résiste à la disparition programmée, symbolique ou matérielle, grâce à divers types de pressions venues «du bas». Mickael Bertrand retrace l'histoire des efforts déployés depuis plusieurs décennies pour insérer le souvenir de victimes des déportations pour motifs d'homosexualité dans des lieux de commémoration des victimes du nazisme. Cet exemple est particulièrement éclairant sur la double fonction assignée à la mémoire : honorer et prévenir.

7 La question de l'imposition du sens et des stratégies développées dans cet objectif par toutes sortes d'acteurs de différente importance, au cœur de toute interrogation sur les phénomènes de mémoire collective, est soulevée de façon plus ou moins explicite par l'ensemble des contributeurs. La répartition des textes en trois parties censées distinguer les lieux de mémoire victimaire en fonction de leur "réussite » redevable à des politiques d'État ou des entreprises privées ou de leur "échec ", montre à quel point cette question du contrôle reste centrale dans toute analyse des représentations collectives. De ce point de vue, l'ouvrage offre là aussi des éclairages très diversifiés. Sophie Lambroschini montre à partir du cas de la Grande famine en Ukraine, comment la mémoire de ce drame, cultivée activement depuis la fin des années 1980, est appropriée par le régime en place après 1991 dans une construction, le Holodomor, destinée à le placer au même niveau que d'autres génocides européens. Kathy Rousselet interroge le rôle que veut jouer l'Église orthodoxe dans la constitution de la mémoire des répressions staliniennes. Piotr H. Kosicki analyse les difficultés à instaurer un lieu de mémoire aux victimes de Katyń malgré l'évolution de la connaissance sur ce drame aussi bien en Pologne qu'en Russie. Il montre comment les contradictions des objectifs poursuivis par les familles des victimes, les acteurs politiques russes ou polonais, entravent ce processus. Florent Brayard propose une analyse particulièrement subtile sur la politique mémorielle nazie, en traquant les indices des stratégies d'effacement de traces. Il s'attaque ainsi au vrai problème de toute "politique mémorielle " : celui des décisions qui la mettent en place, question souvent éludée dans les études sur ce sujet qui se concentrent plus sur l'aval desdites politiques (leurs résultats, ou leurs réalisations) plutôt que sur l'amont ${ }^{1}$ beaucoup plus difficile à restituer.

Au total, nous avons là une somme impressionnante de travaux sur la mémoire qui nous oblige à déplacer constamment notre regard sur des objets différents et alimente de façon très féconde les réflexions sur les phénomènes de présence du passé dans nos sociétés contemporaines et les moyens de les observer. 


\section{NOTES}

1. A ce sujet, Cf.: Sarah Gensburger, "Réflexions autour de la notion de "politique de la mémoire" » in Michel offerlé, Henry Rousso, éds., La fabrique interdisciplinaire, Rennes : Presses Universitaires de Rennes, 2008, p. 133-148. 